\title{
PTPRF as a novel tumor suppressor through deactivation of ERKI/2 signaling in gastric adenocarcinoma
}

This article was published in the following Dove Press journal:

OncoTargets and Therapy

\section{Xiang'an Tian' \\ Chengju Yang ${ }^{2}$ \\ Liguang Yang' \\ Qinli Sun' \\ Naiqing Liu'}

'First Department of General Surgery, Linyi Central Hospital, Linyi 276400, Shandong Province, China; ${ }^{2}$ Second Department of Obstetrics, Linyi Central Hospital, Linyi 276400 , Shandong Province, China
Correspondence: Naiqing Liu First Department of General Surgery, Linyi Central Hospital, 17 Jiankang Road, Linyi Central Hospital, Linyi 276400,

China

Email kangji73666@I63.com
Background: Protein tyrosine phosphatase, receptor type F (PTPRF) is an important phosphatase playing roles in regulating cell growth, differentiation and oncogenic transformation. Overexpression of PTPRF has been observed in non-small cell lung cancer, but its clinical significance in other malignancies is still unknown.

Methods: We explored the expression pattern of PTPRF in gastric adenocarcinoma by using RTqPCR and immunohistochemistry staining. The clinical significance of PTPRF was evaluated by univariate and multivariate analyses. Furthermore, the signaling pathways downstream of PTPRF was investigated by knockdown and overexpression assays combined with cellular studies.

Results: We found a remarkable down-regulation of PTPRF in gastric adenocarcinomas, which was significantly associated with advanced tumor TNM stages. Survival analysis showed that lower PTPRF level indicated a poorer overall survival of gastric adenocarcinoma patients. By conducting knockdown and overexpression studies in gastric adenocarcinoma cells, we revealed the role of PTPRF on inhibiting extracellular signal-regulated kinase-1/2 (ERK1/2) phosphorylation and its downstream signaling. Consistent with clinical findings, cellular results demonstrated that overexpressing PTPRF can significantly inhibit tumor migration and invasion, while silencing PTPRF showed opposite effects.

Conclusion: In conclusion, patients with lower PTPRF expression in gastric adenocarcinoma tissues were more predisposed to advanced tumor stage and unfavorable prognosis.

Keywords: gastric cancer, PTPRF, prognosis, invasion

\section{Introduction}

Gastric adenocarcinoma comprises $90 \%$ of the cancers that occur in the stomach, and is recognized as the second-most common malignancy worldwide. ${ }^{1}$ Despite the great advances in treatment that have been achieved over the past few decades, ${ }^{2}$ patients with unresectable gastric cancer possess a very poor prognosis, and 5-year overall survival (OS) ranges from $8 \%$ to $20 \%{ }^{3}$ As such, there is still much need to illuminate the progression and metastasis mechanisms of gastric adenocarcinoma and identify novel prognostic biomarkers for more effective therapeutic drugs. ${ }^{4}$

Besides protein-abundance alteration, more and more attention is focusing on the effects of protein posttranslational modifications on tumor development, such as ubiquitination and phosphorylation. ${ }^{5,6}$ The phosphorylation balance is regulated by various kinases and phosphatases. PTPRF is a receptor-type protein-tyrosine phosphatase that catalyzes the dephosphorylation of tyrosine residues. ${ }^{7}$ PTPRF is also called leukocyte common antigen-related receptor, due to its initially identified roles in leukemia. ${ }^{8}$ As with many other phosphatases, PTPRF has been reported to participate in cell 
proliferation and differentiation regulation by targeting its downstream substrates. ${ }^{9}$

Of note, PTPRF has been considered to suppress the carcinogenesis of liver cancer and its downregulation to facilitate tumor development. ${ }^{10}$ However, the role of PTPRF seems controversial in breast cancer. For example, PTPRF has shown a positive correlation with tumor metastasis in a mouse model of mammary adenocarcinoma. ${ }^{11}$ In contrast, another study demonstrated the involvement of PTPRF in suppressing breast-tumor-cell metastasis by deactivating EGFR signaling. ${ }^{12}$ Additionally, although high PTPRF expression was found to be prognostic for shorter OS, it is also significantly predictive for an improved survival with erlotinib treatment. ${ }^{13}$ Therefore, the tumor-related role of PTPRF seems highly specific in distinct tumor types.

In the current study, we firstly examined mRNA and protein levels of PTPRF in gastric adenocarcinoma tissue and adjacent normal gastric tissue. Then, we identified a positive correlation between lower PTPRF levels and TNM stages of gastric adenocarcinoma patients. Furthermore, we found that lower PTPRF expression indicated poorer clinical outcomes, and thus identified PTPRF as an independent prognostic factor for gastric adenocarcinoma patients. Finally, cellular studies revealed the direct effects of PTPRF on regulating tumor metastasis and corresponding signaling pathways.

\section{Methods}

\section{Patients and samples}

Formalin-fixed, paraffin-embedded gastric adenocarcinoma tissue together with adjacent normal gastric tissue were obtained from surgery in 115 randomly selected patients during 2008-2014 in Linyi Central Hospital. All patients were followed up for 9-72 months, and 61 had passed away by the end of follow-up. Another 27 pairs of clinical specimens from surgical resection were freshly frozen in liquid nitrogen and stored for further use. All specimens used in the present study were confirmed based on pathology and histology examination. This study was approved by the ethics committee of Linyi Central Hospital. Written informed consent was obtained from all patients.

\section{Real-time quantitative PCR}

Total mRNA was isolated from 27 pairs of gastric adenocarcinoma tissue and adjacent gastric tissue using Trizol reagent (Thermo Fisher Scientific, Waltham, MA, USA). The purity and concentration of isolated mRNA was tested by NanoDrop 2000 at $\mathrm{OD}_{260}$. Exactly $1 \mu \mathrm{g}$ of total $\mathrm{mRNA}$ from each specimen was reverse-transcribed into cDNA using SuperScript cDNA (Thermo Fisher Scientific) according to the manufacturer's instructions. Quantitative PCR was then conducted to evaluate the mRNA level of PTPRF using SYBR green PCR master mix (Thermo Fisher Scientific) with PTPRF primers (forward primer 5'-ATGTCATCGCCTACGACCACTC-3', reverse primer 5'-GTGGCGATGTAGGCATTCTGCT-3'). Gene-expression levels were normalized by $G A P D H$ (forward primer 5'-GTGAAGGTCGGAGTCAACGG-3', reverse primer 5'-TCAATGAAGGGGTCATTGATGG-3') with the $2^{-\triangle \Delta \mathrm{CT}}$ method. ${ }^{14}$

\section{Immunochemistry staining and evaluation}

Immunochemistry (IHC) staining was performed as described by others. ${ }^{15}$ Briefly, $4 \mu \mathrm{m}$ tissue sections were firstly incubated with polyclonal anti-PTPRF antibody (1:300 dilution; Santa Cruz Biotechnology, Dallas, TX, USA) overnight at $4^{\circ} \mathrm{C}$, then incubated with horseradish peroxidase-conjugated secondary antibody for 1 hour at $37^{\circ} \mathrm{C}$. Instead of antiPTPRF antibody, PBS was used as a negative control for IHC assays. After final staining with a DAB-substrate kit (Beyotime Biotechnology, Beijing, China), IHC slides were reviewed by two independent pathologists. IHC scores were evaluated based on both the percentage of positively stained cells and staining intensity. The percentage of positive tumor cells was scored as $1(0 \%-25 \%$ positive tumor cells), $2(25 \%-50 \%$ positive tumor cells), 3 (50\%-75\% positive tumor cells), and 4 ( $>75 \%$ positive tumor cells). Staining intensity was scored as 1 (no staining), 2 (light yellow), 3 (dark yellow), and 4 (brown). The final IHC score was calculated by multiplying the percentage score with intensity score. Among all patients, four were scored 1, four scored 2, 12 scored 3, 16 scored 4, 22 scored 6, 19 scored 8, 16 scored 9, 15 scored 12, and seven scored 16. According to median score, all enrolled patients were classified into two groups: high PTPRF expression (staining score $\geq 8$ ) and low PTPRF expression (staining score $<8$ ).

\section{Cell culture and transfection}

HEK293 cells and the human originated gastric adenocarcinoma cell line MKN45 was purchased from ATCC (Manassas, VA, USA). Normal human gastric epithelial cells were purchased from KeyGen Biotech (Nanjing, Jiangsu, China). All cells were cultured in DMEM supplemented with 10\% (Thermo Fisher Scientific), $100 \mathrm{U} / \mathrm{mL}$ penicillin, and $100 \mathrm{mg} / \mathrm{mL}$ streptomycin. The human PTPRF-coding region was amplified from HEK293 cells using two primers (forward 5'-CCCCGGTACCATGGCCCCTGAGCCAG CC-3', reverse 5'-CCCCGCGGCCGCCCGTTGCATAGTG GTCAAA-3'), ${ }^{12}$ then inserted into a pCDNA3.1 vector. The PTPRF siRNA oligoduplex was synthesized with the 
sequence 5'-CAGCGCTATCTAGATAGGTAA-3'. ${ }^{16}$ Transfection of PTPRF plasmid or siRNA were carried out with Lipofectamine 2000 according to the manufacturer's instructions.

\section{Western blot}

Immunoblotting assays were performed as described by others to evaluate the expression or phosphorylation levels of various proteins. ${ }^{17}$ Briefly, harvested cell pellets were homogenized in NP40 lysis buffer to generate total cell lysates. Total protein concentration was measured using a BCA protein-assay kit (Thermo Fisher Scientific), and $20 \mu \mathrm{g}$ total protein was subjected onto $10 \%$ SDS/PAGE gels, transferred onto polyvinylidene difluoride membranes (EMD Millipore, Billerica, MA, USA), blocked with 5\% nonfat milk, and incubated with primary antibodies (Santa Cruz Biotechnology). Horseradish peroxidase-conjugated secondary antibodies were then incubated for 1 hour at room temperature, followed by detection using chemiluminescence solution and X-ray film.

\section{Proliferation, migration, and invasion assays} Cell proliferation was examined using the MTT assay. ${ }^{18}$ Briefly, $5 \times 10^{3}$ cells were added to $96-$ well plates and cultured for different times. MTT solution was added to each well and incubated for 4 hours at $37^{\circ} \mathrm{C}$, followed by measurement of $\mathrm{OD}_{490}$ absorbance using the automated plate reader. Migration and invasion capacity was measured by transwell assays. ${ }^{15}$ For the invasion assay, the transwell was precoated with Matrigel as described by others. ${ }^{19}$ Briefly, $3 \times 10^{4}$ cells were added to the upper chamber and cultured for 48 hours. Migrated or invaded cells were fixed and stained. Cell counting was carried out in five random visual fields. All experiments were performed in triplicate and repeated independently at least three times.

\section{Statistics}

All statistical analyses were performed using SPSS 24.0. Correlations between expression levels of PTPRF and patient characteristics were tested with $\chi^{2}$ or Fisher's exact tests. Survival analyses were conducted by the Kaplan-Meier method and compared with log-rank tests. Multivariate Cox regression analysis was used to identify independent prognostic factors using a forward stepwise approach. For cellular experiments, data are presented as means \pm SEM from three independent experiments and compared using Student's $t$-test. $P<0.05$ by two-tail criteria was considered statistically significant.

\section{Results \\ Patient information}

The entire cohort contained 75 males and 40 females, with a median age of 53 years. A total of 48 patients had larger tumors (diameter $>5.0 \mathrm{~cm}$ ), while tumor diameter for the other 67 patients was $<5 \mathrm{~cm}$ (Table 1). Ten patients were diagnosed with good pathological differentiation, 53 moderate differentiation, and the other 52 poor differentiation. Additionally, we retrieved the tumor locations of gastric adenocarcinomas: 22 patients had tumors at upper gastric level, while the other 93 had tumors at the lower gastric level. In general, 47 patients were classified as TNM stage I/II and the other 68 TNM stage III/IV.

\section{PTPRF is downregulated in gastric adenocarcinoma tissue}

We firstly tested mRNA abundance in both gastric adenocarcinoma tissue and adjacent normal tissue, which revealed decreased PTPRF mRNA levels in tumor tissue (Figure 1A, $P=0.011$ ). Secondly, we compared protein levels of PTPRF in normal GES1 gastric epithelial cells with MKN45 cells. As expected, PTPRF showed lower protein levels in MKN45 cells (Figure 1B). We also searched the Cancer Genome Atlas database and found that lower PTPRF mRNA levels indicated poorer OS of gastric adenocarcinoma patients (Figure 1C, $P=0.003$ ). We next tested the protein expression of PTPRF in clinical specimens. IHC results showed membrane and

Table I Correlations between PTPRF expression and patient features

\begin{tabular}{|c|c|c|c|c|}
\hline \multirow[t]{2}{*}{ Factors } & \multirow{2}{*}{$\begin{array}{l}\text { Cases } \\
(n=I \mid 5)\end{array}$} & \multicolumn{2}{|c|}{ PTPRF level } & \multirow[t]{2}{*}{$P$-value } \\
\hline & & $\begin{array}{l}\text { Low } \\
(n=58)\end{array}$ & $\begin{array}{l}\text { High } \\
(n=57)\end{array}$ & \\
\hline Age & & & & 0.790 \\
\hline$\leq 50$ years & 45 & 22 & 23 & \\
\hline$>50$ years & 70 & 36 & 34 & \\
\hline Sex & & & & 0.395 \\
\hline Female & 40 & 18 & 22 & \\
\hline Male & 75 & 40 & 35 & \\
\hline Tumor size & & & & 0.070 \\
\hline$\leq 5 \mathrm{~cm}$ & 67 & 29 & 38 & \\
\hline$>5 \mathrm{~cm}$ & 48 & 29 & 19 & \\
\hline Differentiation & & & & 0.299 \\
\hline Good/moderate & 63 & 29 & 34 & \\
\hline Poor & 52 & 29 & 23 & \\
\hline Localization & & & & $0.096^{\mathrm{a}}$ \\
\hline Upper gastric & 22 & 15 & 7 & \\
\hline Lower gastric & 93 & 43 & 50 & \\
\hline TNM stage & & & & $0.00 I^{*}$ \\
\hline $\mathrm{I} / \mathrm{II}$ & 47 & 15 & 32 & \\
\hline III/IV & 68 & 43 & 25 & \\
\hline
\end{tabular}

Notes: aFisher's exact test, due to limited cases; ${ }^{* P}<0.05\left(\chi^{2}\right)$. 


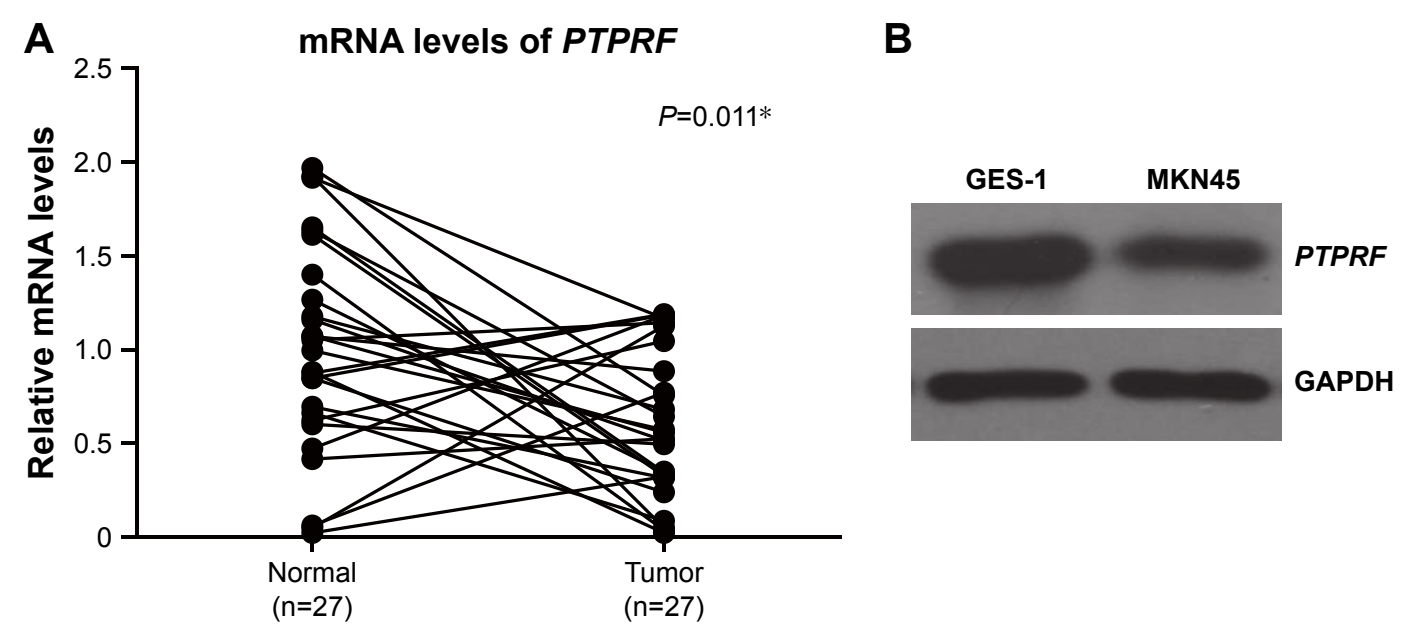

\section{Effect of PTPRF mRNA level on gastric adenocarcinoma patient survival}
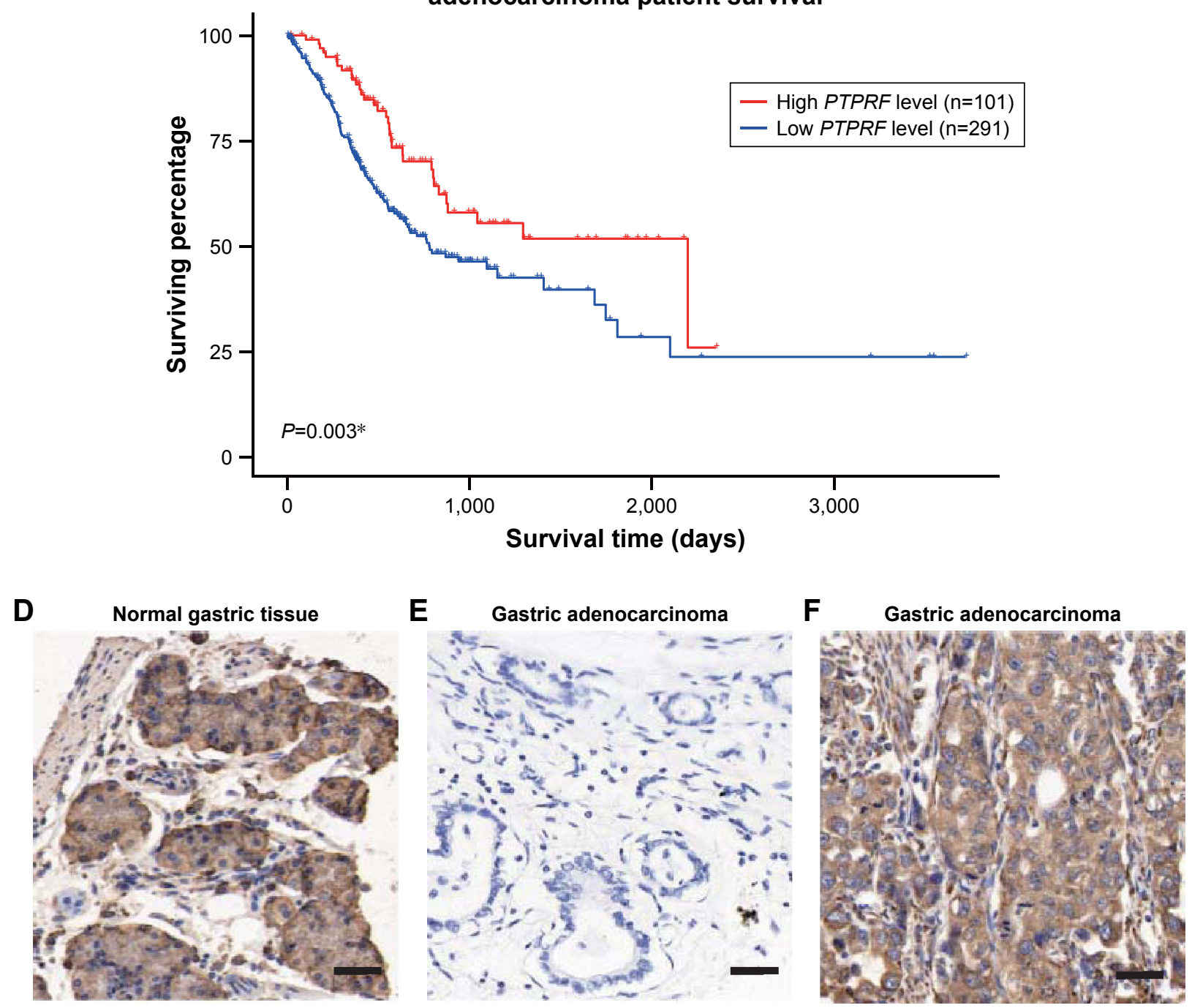

Figure I PTPRF is downregulated in gastric adenocarcinoma tissue.

Notes: (A) Real-time PCR quantification of PTPRF mRNA levels in gastric adenocarcinoma tissue and matched adjacent nontumoral tissue ( $\mathrm{n}=27)$. The $m R N A$ expression of PTPRF in gastric adenocarcinoma tissue was significantly lower than in nontumoral tissue. (B) PTPRF showed a lower protein level in MKN45 tumor cells than normal GESI epithelial cells. (C) Effect of PTPRF mRNA level on the overall survival of gastric adenocarcinoma patients (data generated from Cancer Genome Atlas database), showing its possible antitumor roles. Representative immunohistochemistry (IHC) results of PTPRF protein expression in adjacent nontumoral tissue (D) and gastric adenocarcinoma tissue (E), showing positive staining in both nucleus and cytoplasm. (F) Representative high expression of PTPRF in gastric adenocarcinoma tissue (IHC score I2). $* P<0.05$ (paired Student's $t$-test); bar $100 \mu \mathrm{m}$. 
cytoplasm localization of PTPRF in normal gastric tissue (Figure 1D), with much lower or negative expression in gastric adenocarcinoma tissue (Figure 1E). Representative high expression of PTPRF protein in tumor tissue is shown in Figure 1F.

\section{Decreased PTPRF is correlated with advanced tumor stage}

According to the defined scoring and classification criteria, we divided enrolled gastric adenocarcinoma patients into a PTPRF low-expression group $(n=58)$ and PTPRF highexpression group $(n=57)$ based on immunoreactivity. Then, we evaluated correlations of PTPRF expression with clinicopathological features in gastric adenocarcinoma patients (Table 1). We found that the lower levels of PTPRF expression were significantly correlated with advanced TNM stages $(P=0.001)$. Additionally, patients with larger tumors also exhibited lower PTPRF levels, though this did not reach statistical significance $(P=0.070)$. No associations were observed between PTPRF expression and age, sex, tumor differentiation, or tumor location $(P>0.05)$. Statistical associations between PTPRF with
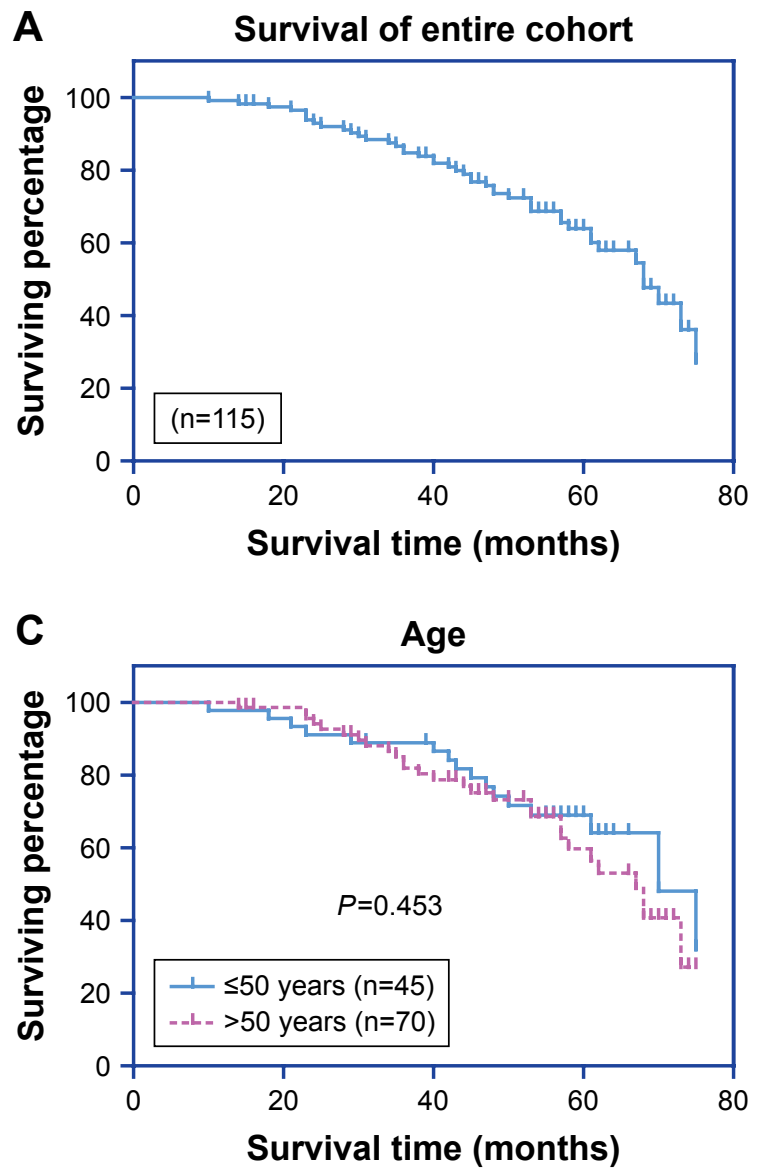

Figure 2 (Continued) tumor stages revealed its possible antitumor effects in gastric adenocarcinoma.

\section{Lower PTPRF protein level indicates poorer clinical outcomes}

The 5-year OS rate of our entire cohort was $60.09 \%$ (Figure 2A). The effect of each clinicopathological characteristic was also evaluated by Kaplan-Meier survival curves (Figure 2B and $\mathrm{H}$ ). With univariate regression analysis, we identified that lower PTPRF protein expression was an unfavorable parameter affecting patient survival (mean survival $52.2 \pm 2.8$ vs $66.4 \pm 1.8$ months, $P<0.001$; Table 2 ), further confirming the association between PTPRF and tumor progression. Consistently with conventional concepts, tumor TNM stage was also significantly predictive for OS $(P=0.001)$.

To explore the clinical significance of PTPRF further, we put all factors with $P<0.01$ by univariate analysis into a multivariate Cox regression model (Table 3). Among these, advanced TNM stages led to significantly worse prognosis (HR 2.447, 95\% CI 1.163-5.151; $P=0.018$ ). Of note, PTPRF also acted as an independent prognostic factor in our cohort
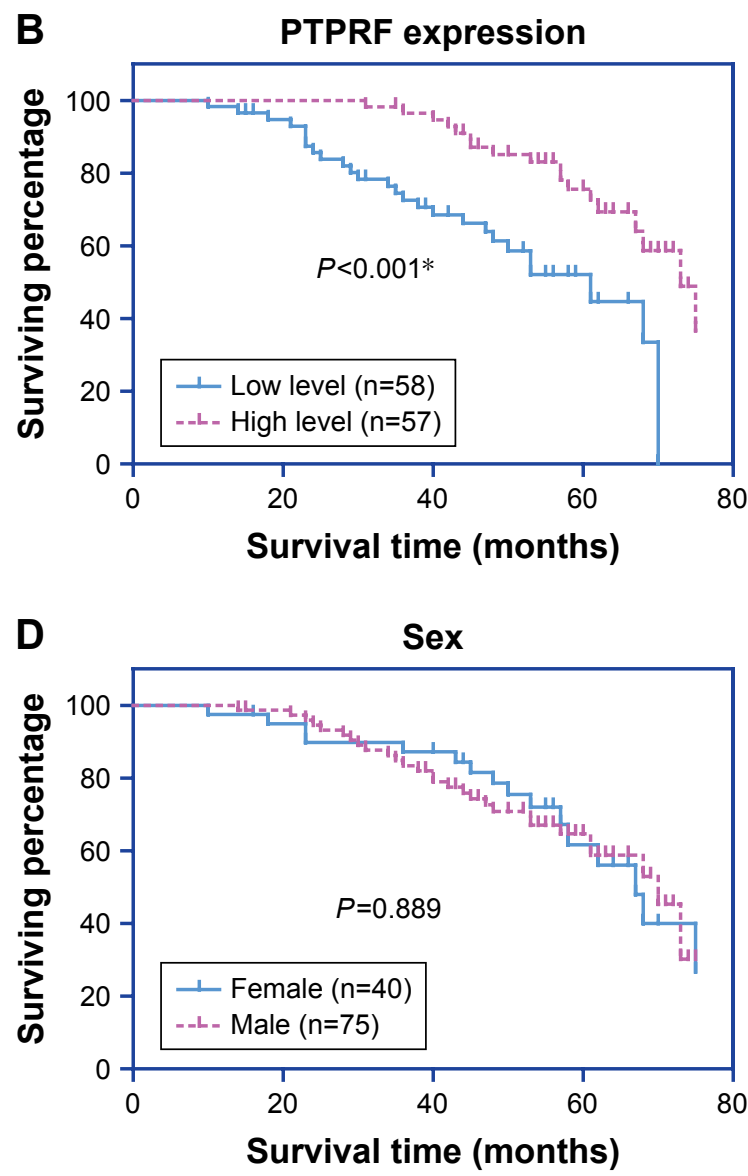

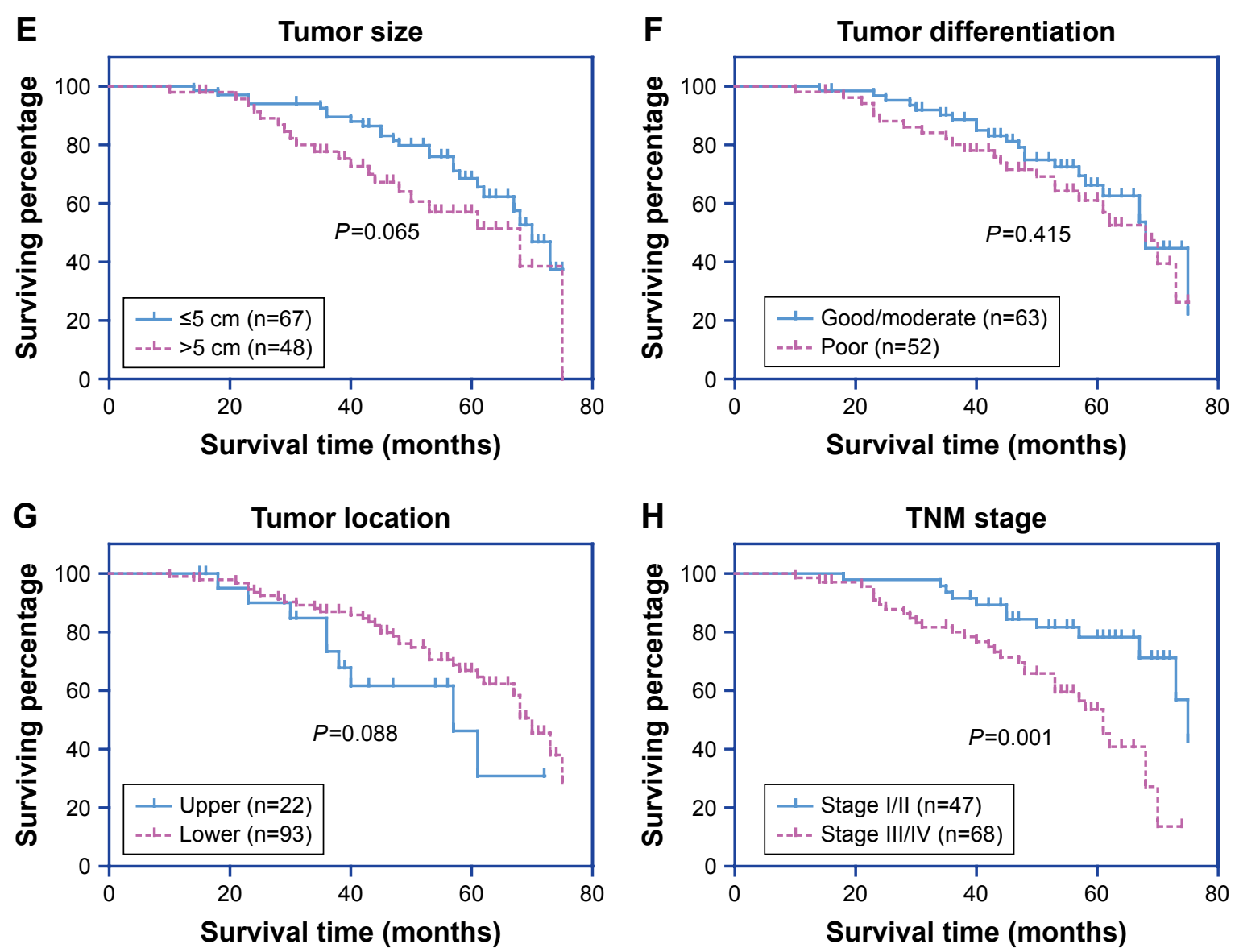

Figure 2 Analysis of overall survival in gastric adenocarcinoma patients.

Notes: The overall survival curve was assessed by the Kaplan-Meier method (A). The effect of each variable was also evaluate: PTPRF level (B), patient age (C), sex (D), tumor size (E), tumor differentiation (F), tumor location $(\mathbf{G})$, and TNM stage $(\mathbf{H})$. $* P<0.05$ (log-rank test).

Table 2 Kaplan-Meier OS analyses

\begin{tabular}{l|l|l|l}
\hline Factors & $\begin{array}{l}\text { OS, months } \\
\text { (mean } \pm \text { SD) }\end{array}$ & $\begin{array}{l}\text { 5-year } \\
\text { OS (\%) }\end{array}$ & P-value \\
\hline Age & $61.7 \pm 3.0$ & 69.0 & 0.453 \\
$\leq 50$ years & $59.2 \pm 2.3$ & 59.7 & 0.889 \\
$>50$ years & $60.4 \pm 3.2$ & 61.6 & \\
Sex & $60.0 \pm 2.3$ & 64.7 & 0.065 \\
Female & $62.8 \pm 2.1$ & 68.5 & \\
Male & $56.3 \pm 3.3$ & 57.1 & \\
Tumor size & $61.9 \pm 2.4$ & 66.3 & 0.415 \\
$\leq 5$ cm & $57.8 \pm 2.8$ & 61.0 & \\
$>5$ cm & $52.7 \pm 4.4$ & 46.2 & 0.088 \\
Differentiation & $61.4 \pm 2.0$ & 66.9 & \\
Good/moderate & & 78.3 & $0.00 I^{\mathrm{a}}$ \\
Poor & $66.8 \pm 2.3$ & 53.6 & \\
Localization & $54.6 \pm 2.4$ & 52.1 & $<0.00 \mathrm{I}^{\mathrm{a}}$ \\
Upper gastric & $52.2 \pm 2.8$ & 75.6 & \\
Lower gastric & $66.4 \pm 1.8$ &
\end{tabular}

Note: a $P 0.05$ (log-rank test).

Abbreviation: OS, overall survival.
(HR 0.492, 95\% CI 0.252-0.959; $P=0.037$ ), indicating that PTPRF exerted antitumor effects in gastric adenocarcinoma. Tumor size or location showed no statistical significance in terms of multivariate analysis.

\section{PTPRF suppresses gastric adenocarcinoma progress by inhibiting ERK signaling}

To determine the underlying mechanisms of PTPRF in suppressing tumor progression, we tested the signaling effects of overexpressing or silencing PTPRF in MKN45 cells. Western blotting results showed that ERK phosphorylation was negatively regulated by PTPRF level (Figure 3A). Consistently, phosphorylation levels of ERK downstream substrates,

Table 3 Multivariate analysis

\begin{tabular}{l|l|l|l}
\hline Factors & HR & $\mathbf{9 5 \%} \mathbf{C I}$ & P-value \\
\hline Tumor size ( $>5$ vs $\leq 5 \mathrm{~cm})$ & $\mathrm{I} .525$ & $0.830-2.80 \mathrm{I}$ & 0.174 \\
Localization (lower vs upper) & 0.477 & $0.222-1.024$ & 0.058 \\
TNM stage (III/IV vs I/II) & 2.447 & $\mathrm{I} .163-5.15 \mathrm{I}$ & $0.018^{\mathrm{a}}$ \\
PTPRF (high vs low) & 0.492 & $0.252-0.959$ & $0.037^{\mathrm{a}}$ \\
\hline
\end{tabular}

Note: ${ }^{a} \mathrm{P}<0.05$ (Cox regression test). 


\section{A}

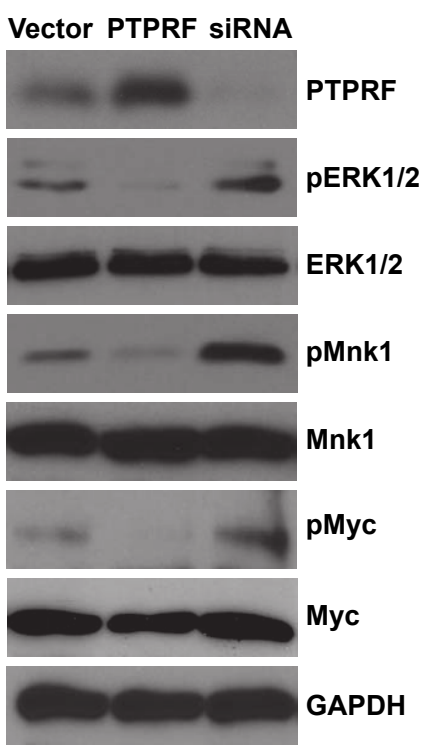

C

Transwell

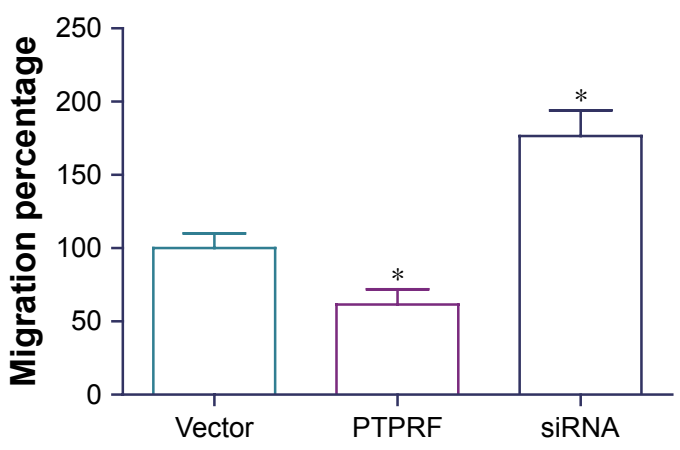

E

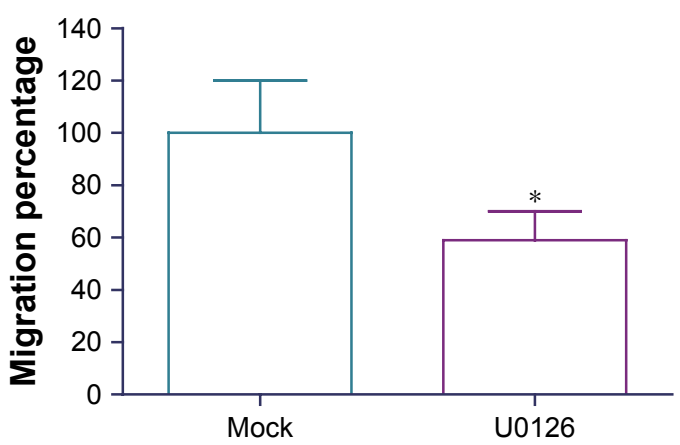

B

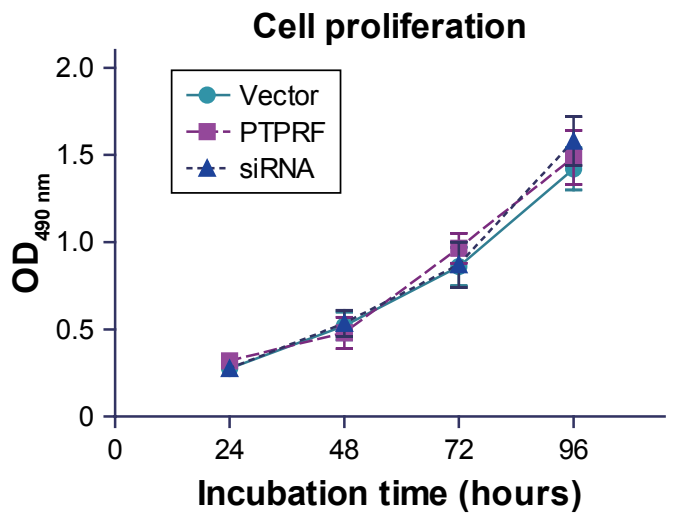

D Matrigel-transwell

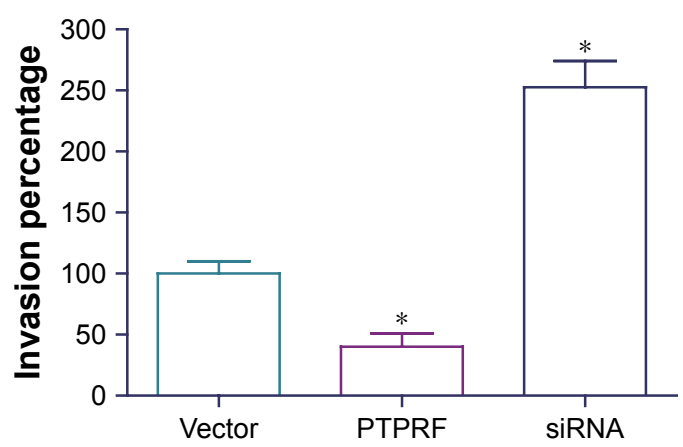

F Matrigel-transwell

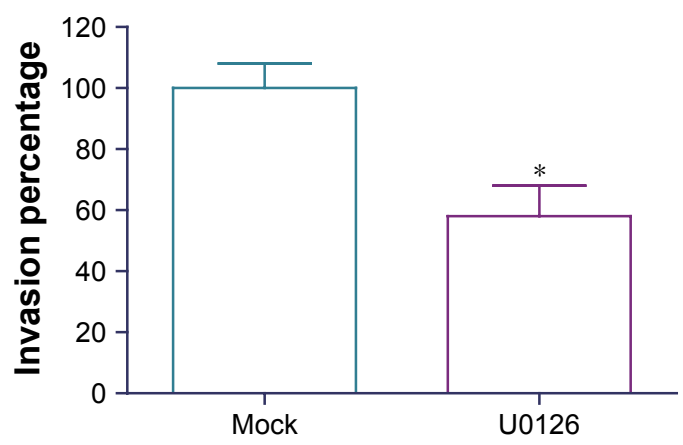

Figure 3 PTPRF suppresses gastric adenocarcinoma progression by downregulating ERK activation.

Notes: (A) Western blot results showed that overexpressing PTPRF downregulated ERK-phosphorylation level. The activity of ERK downstream effectors, such as Mnkl and Myc, was also inhibited by PTPRF. Silencing PTPRF exhibited opposite effects. (B) The proliferation of gastric adenocarcinoma cells was not affected by PTPRF, as revealed by MTT experiments $(P>0.05)$. (C) The migration process of gastric adenocarcinoma cells was tested by transwell assays, revealing a negative correlation between PTPRF level and cell-migration capacity. (D) Matrigel-coated transwell chambers were used to evaluate the invasion process of gastric adenocarcinoma cells, which demonstrated that PTPRF knockdown promoted cell invasion whereas PTPRF overexpression inhibited cell invasion. Similarly, the migration (E) and invasion (F) capacities of MKN45 cells were both inhibited by U0I26, a specific ERK inhibitor. $* P<0.05$ (Student's $t$-test).

such as Mnk1 and Myc, were also significantly inhibited by PTPRF. Since Mnk1 and Myc are well-known tumorrelated proteins, ${ }^{20,21}$ we next tested whether PTPRF could modulate the phenotypes of MKN45 cells. Cell-proliferation assays identified little effect of PTPRF on cell growth (Figure 3B), which is consistent with clinical data. However, cell-migration and -invasion capacity was significantly promoted by silencing PTPRF, while PTPRF overexpression 
inhibited cell migration and invasion (Figure 3C and D). Consistently with the effect of PTPRF, the ERK inhibitor U0126 also significantly impaired the migration and invasion capacity of MKN45 cells (Figure 3E and F).

\section{Discussion}

Gastric adenocarcinoma is the most common type of gastric cancer, and our data showed an antitumor effect of PTPRF on gastric adenocarcinoma for the first time. The role of PTPRF in gastric adenocarcinoma is consistent with previous findings about its effects on breast cancer progression. ${ }^{12}$ The authors demonstrated that PTPRF can downregulate the phosphorylation level of EGFR, which is a tumor-promoting membrane receptor in many tumor types. However, they did not provide the exact phosphorylation sites on EGFR. Additionally, their findings showed consistent alterations of EGFR downstream effectors, including MMP2, MMP11, and ERK. In contrast, another study showed completely opposite effects of PTPRF in a mouse model of breast cancer, showing that PTPRF promoted tumor metastasis. ${ }^{11}$ Their data also showed cross talk between PTPRF and estrogen receptors. Besides the controversial effects on breast cancer, PTPRF also plays complicated roles in non-small-cell lung cancer. On one hand, high PTPRF protein expression in tumor tissue indicates poorer OS. On the other hand, high PTPRF is positively correlated with better chemotherapy response for the non-small-cell lung cancer patients. ${ }^{13}$ Therefore, more studies are needed to dig further into the mechanisms of PTPRF in different tumor types.

Here, we reported the antitumor effects of PTPRF in gastric adenocarcinoma, which can inhibit tumor-cell migration and invasion by downregulating the ERK-signaling pathway. Inhibition of ERK activation, such as by using the chemical inhibitor U0126, has been reported to downregulate the migration and invasion processes of multiple cell types. ${ }^{22,23}$ Here, we also confirmed its similar anti-invasion role on MKN45 cells, which is consistent with the effect induced by PTPRF. Since MKN45 exhibits positive expression of EGFR and PDGFR proteins, ${ }^{24,25}$ it is possible that PTPRF inhibits gastric adenocarcinoma progression by attenuating growth-factor effects. In addition, the role of PTPRF in inhibiting ERK phosphorylation has been reported in HepG2 liver cancer cells; however, the authors believed that was an indirect effect of regulating ERK-upstream Src and PP2A proteins. ${ }^{26,27}$ Whether PTPRF can directly modulate the phosphorylation status of ERK and Mnk1 needs further experimental evidence.

The major limitation of our study is that all the patients were enrolled from a single medical center, and thus there may have been regional or racial bias. Another limitation is that all the data were obtained from in vitro studies. More evidence on animal models would better complement our findings. However, we at least revealed the protective and predictive effects of PTPRF on gastric adenocarcinoma.

Malignant phenotypes of tumor cells are largely characterized by their migration and invasion capacity. ${ }^{28}$ One tumortreatment strategy is inhibition of metastasis activity. ${ }^{29,30}$ Therefore, our findings on the effect of PTPRF in gastric adenocarcinoma suggest its potential role as a therapeutic target. Taking into consideration the role of PTPRF in sensitizing chemotherapy toward lung cancer, whether PTPRF can help treat gastric adenocarcinoma also deserves further investigation.

\section{Conclusion}

In summary, our data provide the first evidence that PTPRF expression is downregulated in gastric adenocarcinoma. We also explored the clinical value of PTPRF in gastric adenocarcinoma patients, such as in predicting OS. In addition, this study showed that PTPRF can suppress gastric cancer-cell migration and invasion, perhaps by inhibiting ERK signaling, revealing its potential in therapeutic development.

\section{Disclosure}

The authors report no conflicts of interest in this work.

\section{References}

1. Japanese Gastric Cancer Association. Japanese gastric cancer treatment guidelines 2014 (ver. 4). Gastric Cancer. 2017;20(1):1-19.

2. Harada K, Mizrak Kaya D, Shimodaira Y, Ajani JA. Global chemotherapy development for gastric cancer. Gastric Cancer. 2017;20(Suppl 1): 92-101.

3. Siegel RL, Miller KD, Jemal A. Cancer statistics, 2018. CA Cancer J Clin. 2018;68(1):7-30.

4. Zhang Q, Liu H, Zhu Q, et al. Patterns and functional implications of platelets upon tumor "education". Int J Biochem Cell Biol. 2017; 90:68-80.

5. Liu H, Zhang Q, Li K, et al. Prognostic significance of USP33 in advanced colorectal cancer patients: new insights into $\beta$-arrestindependent ERK signaling. Oncotarget. 2016;7(49):81223-81240.

6. Xu Y, Yang X, Li Z, et al. Sprouty2 correlates with favorable prognosis of gastric adenocarcinoma via suppressing FGFR2-induced ERK phosphorylation and cancer progression. Oncotarget. 2017;8(3):4888.

7. Ahmad F, Goldstein BJ. Functional association between the insulin receptor and the transmembrane protein-tyrosine phosphatase LAR in intact cells. J Biol Chem. 1997;272(1):448-457.

8. Schaapveld RQ, van den Maagdenberg AM, Schepens JT, et al. The mouse gene Ptprf encoding the leukocyte common antigen-related molecule LAR: cloning, characterization, and chromosomal localization. Genomics. 1995;27(1):124-130.

9. Chagnon MJ, Uetani N, Tremblay ML. Functional significance of the LAR receptor protein tyrosine phosphatase family in development and diseases. Biochem Cell Biol. 2004;82(6):664-675.

10. Bera R, Chiou CY, Yu MC, et al. Functional genomics identified a novel protein tyrosine phosphatase receptor type F-mediated growth inhibition in hepatocarcinogenesis. Hepatology. 2014;59(6):2238-2250. 
11. Levea CM, McGary CT, Symons JR, Mooney RA. PTP LAR expression compared to prognostic indices in metastatic and non-metastatic breast cancer. Breast Cancer Res Treat. 2000;64(2):221-228.

12. Du WW, Fang L, Li M, et al. MicroRNA miR-24 enhances tumor invasion and metastasis by targeting PTPN9 and PTPRF to promote EGF signaling. $J$ Cell Sci. 2013;126(Pt 6):1440-1453.

13. Soulières D, Hirsch FR, Shepherd FA, et al. PTPRF Expression as a Potential Prognostic/Predictive Marker for Treatment with Erlotinib in NonSmall-Cell Lung Cancer. J Thorac Oncol. 2015;10(9):1364-1369.

14. Tan W, Pan M, Liu H, Tian H, Ye Q, Liu H. Ergosterol peroxide inhibits ovarian cancer cell growth through multiple pathways. Onco Targets Ther. 2017;10:3467-3474.

15. Liu H, Xu Y, Zhang Q, et al. Prognostic significance of TBL1XR1 in predicting liver metastasis for early stage colorectal cancer. Surg Oncol. 2017;26(1):13-20.

16. Martin KR, Xu Y, Looyenga BD, et al. Identification of PTPsigma as an autophagic phosphatase. J Cell Sci. 2011;124(Pt 5):812-819.

17. Zhang Q, Yuan L, Liu D, et al. Hydrogen sulfide attenuates hypoxiainduced neurotoxicity through inhibiting microglial activation. Pharmacol Res. 2014;84:32-44.

18. Guo S, Liu HD, Liu YF, Liu L, Sun Q, Cui XJ. Hepatoma-derived growth factor: a novel prognostic biomarker in intrahepatic cholangiocarcinoma. Tumour Biol. 2015;36(1):353-364.

19. Liu H, Liu Z, Li K, et al. TBL1XR1 predicts isolated tumor cells and micrometastasis in patients with TNM stage I/II colorectal cancer. J Gastroenterol Hepatol. 2017;32(9):1570-1580.

20. Hou S, Du P, Wang P, Wang C, Liu P, Liu H. Significance of MNK1 in prognostic prediction and chemotherapy development of epithelial ovarian cancer. Clin Transl Oncol. 2017;19(9):1107-1116.
21. Dang CV, Le A, Gao P. MYC-induced cancer cell energy metabolism and therapeutic opportunities. Clin Cancer Res. 2009;15(21):6479-6483.

22. Malik A, Pal R, Gupta SK. Interdependence of JAK-STAT and MAPK signaling pathways during EGF-mediated HTR-8/SVneo cell invasion. PLoS One. 2017;12(5):e178269.

23. Xiao G, Wang X, Wang J, et al. CXCL16/CXCR6 chemokine signaling mediates breast cancer progression by pERK1/2-dependent mechanisms. Oncotarget. 2015;6(16):14165.

24. Wang J, Yang S, Cai X, et al. Berberine inhibits EGFR signaling and enhances the antitumor effects of EGFR inhibitors in gastric cancer. Oncotarget. 2016;7(46):76076.

25. Wang JX, Zhou JF, Huang FK, et al. GLI2 induces PDGFRB expression and modulates cancer stem cell properties of gastric cancer. Eur Rev Med Pharmacol Sci. 2017;21(17):3857-3865.

26. Huang Y, Zhang Y, Ge L, Lin Y, Kwok HF. The Roles of Protein Tyrosine Phosphatases in Hepatocellular Carcinoma. Cancers. 2018; 10(3):82.

27. Bera R, Chiou CY, Mc Y, et al. Functional genomics identified a novel protein tyrosine phosphatase receptor type f-mediated growth inhibition in hepatocarcinogenesis. Hepatology. 2014;59(6):2238-2250.

28. Fan Q, Liu B. Identification of a RNA-Seq Based 8-Long Non-Coding RNA Signature Predicting Survival in Esophageal Cancer. Med Sci Monit. 2016;22:5163-5172.

29. Alowayed N, Salker MS, Zeng N, Singh Y, Lang F. LEFTY2 Controls Migration of Human Endometrial Cancer Cells via Focal Adhesion Kinase Activity (FAK) and miRNA-200a. Cell Physiol Biochem. 2016; 39(3):815-826.

30. Duan J, Huang W, Shi H. Positive expression of KIF20A indicates poor prognosis of glioma patients. Onco Targets Ther. 2016;9:6741-6749.
OncoTargets and Therapy

\section{Publish your work in this journal}

OncoTargets and Therapy is an international, peer-reviewed, open access journal focusing on the pathological basis of all cancers, potential targets for therapy and treatment protocols employed to improve the management of cancer patients. The journal also focuses on the impact of management programs and new therapeutic agents and protocols on

\section{Dovepress}

patient perspectives such as quality of life, adherence and satisfaction. The manuscript management system is completely online and includes a very quick and fair peer-review system, which is all easy to use. Visit http://www.dovepress.com/testimonials.php to read real quotes from published authors. 